Published in final edited form as:

Sleep Health. 2016 December ; 2(4): 289-296. doi:10.1016/j.sleh.2016.08.004.

\title{
Age differences in workplace intervention effects on employees' nighttime and daytime sleep
}

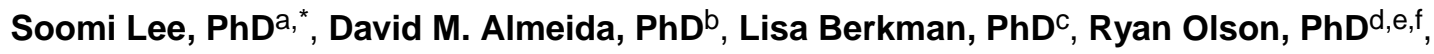 \\ Phyllis Moen, PhDg, and Orfeu M. Buxton, PhD ${ }^{a, h, i, j}$ \\ aDepartment of Biobehavioral Health, Pennsylvania State University, University Park, PA \\ bDepartment of Human Development and Family Studies, Pennsylvania State University, \\ University Park, PA \\ ${ }^{\mathrm{C} C e n t e r}$ for Population and Development Studies, Harvard University, Cambridge, MA \\ dOregon Institute of Occupational Health Sciences, Oregon Health \& Science University, Portland, \\ OR \\ eSchool of Public Health, Oregon Health \& Science University/Portland State University, Portland, \\ OR \\ fDepartment of Psychology, Portland State University, Portland, OR \\ gDepartment of Sociology, University of Minnesota, Minneapolis, MN \\ hDivision of Sleep Medicine, Harvard Medical School, Boston, MA \\ iDepartment of Social and Behavioral Sciences, Harvard Chan School of Public Health, Boston, \\ MA
}

iDepartment of Medicine, Brigham and Women's Hospital, Boston, MA

\begin{abstract}
Objectives-To examine the effects of a workplace flexibility/support intervention on employees' sleep quantity and quality during nights and days and whether the effects differ by employee age.
\end{abstract}

Design-Cluster-randomized controlled trial.

Setting-Information technology industry workplaces.

\footnotetext{
*Corresponding author at: Department of Biobehavioral Health, The Pennsylvania State University, 401 Biobehavioral Health Building, University Park, PA 16802-650. Tel.: +1 814880 4344. smlee@psu.edu, soomilee1104@ gmail.com (S. Lee).

Disclosure

This was not an industry supported study. This research was conducted as part of the Work, Family, and Health Network (www.WorkFamilyHealthNetwork.org), which is funded by a cooperative agreement through the National Institutes of Health and the Centers for Disease Control and Prevention: Eunice Kennedy Shriver National Institute of Child Health and Human Development (U01HD051217, U01HD051218, U01HD051256, U01HD051276), National Institute on Aging (U01AG027669), Office of Behavioral and Social Sciences Research, and National Institute for Occupational Safety and Health (U01OH008788,

U01HD059773). Grants from the National Heart, Lung and Blood Institute (R01HL107240), the William T. Grant Foundation, Alfred P Sloan Foundation, and the Administration for Children and Families provided additional funding. The contents of this publication are solely the responsibility of the authors and do not necessarily represent the official views of these institutes and offices. No offlabel or investigational use is indicated. The authors have indicated no financial conflicts of interest.
} 
Participants-US employees ( $M_{\mathrm{age}}=46.9$ years) at an information technology firm who provided actigraphy at baseline and a 12-month follow-up $(\mathrm{N}=396 ; \mathrm{n}=195$ intervention, $\mathrm{n}=201$ control).

Intervention-The Work, Family, and Health Study intervention aimed to increase workplace flexibility and support. The intervention consisted of facilitated discussions to help employees increase control over when and where they work as well as manager-specific training sessions to increase manager support for employees' work-family issues.

Measurements-Nighttime sleep duration, wake after sleep onset (WASO), and nap duration were measured with wrist actigraphy. Day-to-day variability in these variables $\left(\mathrm{min}^{2}\right)$ was also estimated.

Results-Intervention employees increased nighttime sleep duration at 12 months, by 9 minutes per day, relative to control employees. There were interaction effects between the intervention and age on daytime nap duration and day-to-day variability in WASO. Older employees (56-70 years) in the intervention condition decreased nap duration at 12 months relative to older employees in the control condition. Older employees in the intervention condition also exhibited a greater decrease in day-to-day variability of WASO at 12 months compared with their baseline.

Conclusions-The workplace flexibility/support intervention was effective in enhancing employees' sleep health by increasing nighttime sleep duration. Furthermore, the intervention was particularly effective for older employees in decreasing their daytime nap duration and day-to-day variability in WASO.

\section{Keywords}

Nighttime sleep duration; Wake after sleep onset; Nap duration; Workplace intervention; Day-today variability in sleep

Sleep deficiency is an increasing concern in the US workforce. In 2012, approximately $30 \%$ of US workers reported that they sleep less than 6 hours per night. ${ }^{1}$ Recent research shows that men and women who have a short sleep duration, especially less than 6 hours, are at increased risk for disease compared with those who get 7 to 9 hours of sleep. ${ }^{2}$ However, getting sufficient hours for sleep is challenging in our technology-driven society where employees may be available 24/7. Indeed, technological advances and other societal changes have increased work hours, ${ }^{3}$ which has decreased workers' sleep time. ${ }^{4}$ Sleep deficiency, such as short nighttime sleep duration and disruptions of sleep quality, leads to excessive daytime sleepiness for many workers, which in turn increase risks for workplace injuries and reduced productivity. ${ }^{5}$

This study examined the effects of a workplace intervention with predominantly dayshift workers on changes in sleep quantity and quality during nights and days. There is little research examining the effects of a workplace intervention on employee sleep, except for a few studies reporting on an intervention that promoted retail employees' work-time flexibility increasing self-reported sleep duration ${ }^{6}$ and perceptions of sleep sufficiency. ${ }^{7}$ To date, only one study reported a positive effect of a workplace intervention on actigraphically assessed sleep. ${ }^{10}$ This workplace intervention, termed STAR (short for "Support-Transform- 
Achieve-Results"), was implemented in an information technology (IT) firm where the working conditions involve long work hours and high work-to-family conflict (ie, the extent that work stressors interfere with activities in family and personal domains). ${ }^{9,10}$ The intervention was designed to decrease work-to-family conflict by increasing workplace flexibility and support within a randomized control design. As intended, the intervention resulted in decreased work-to-family conflict, ${ }^{9}$ and more importantly, increased actigraphically-assessed total sleep duration (by 8.2 minutes) and perceived sleep sufficiency of intervention employees. ${ }^{8}$ However, the previous analyses did not separately assess nighttime sleep and daytime napping, which limits the ability to specify when and during which sleep periods the intervention achieved its effects. Identifying specific sleep periods that are modifiable by a workplace intervention is important for future workplace intervention strategies to enhance the design and efficiency of work-place wellness programs. In this study, we posit that employees who received the STAR intervention may have increases in their nighttime sleep quantity and quality via reduced work-to-family conflict. Research examining intervention effects on employees' daytime napping in their own naturalistic setting is limited, ${ }^{11}$ but improved nighttime sleep quantity and quality may have implications for reduced need for daytime napping. Therefore, the first aim of this analysis was to evaluate the effects of the STAR intervention on IT employees' changes in nighttime sleep duration and wake after sleep onset (WASO), and daytime nap duration. We hypothesized that the intervention would increase nighttime sleep duration, decrease WASO (improve sleep quality), and reduce daytime napping.

Another aim was to consider that the effects of the intervention on nighttime sleep and daytime napping would vary between older and younger employees. Age-related differences in sleep need and sleep physiology ${ }^{12}$ along with variable psychosocial contexts for workers at different stages of the life course could modify intervention effects. As several aspects of work capacity (eg, working fast, using new technology) typically decrease with age, ${ }^{13,14}$ age-related imbalance between work demands and work capacity may result in older employees' increased need for nighttime sleep recovery. ${ }^{15}$ However, nighttime sleep quantity generally decreases with age through the working years, ${ }^{16}$ possibly mitigating intervention effects on nighttime sleep duration. Age-related deterioration in nighttime sleep quality (eg, more WASO) may entail more daytime napping for older employees than younger employees. For example, when pooled across employment statuses, older individuals have longer total amount of WASO and greater frequency of napping compared with younger individuals. ${ }^{16-20}$ These findings suggest that the effects of the workplace intervention on WASO and nap duration may be more apparent for older employees who have greater need for sleep recovery but "less efficient" sleep physiology than younger counterparts. Thus, we hypothesized that the positive effects of the intervention on WASO and daytime nap duration would be more apparent for older employees than younger employees.

This article also assessed whether a workplace intervention can change day-to-day variability in sleep, in addition to daily amount of sleep. A great degree of variability in sleep may indicate irregular circadian rhythms and sleep patterns, ${ }^{21}$ potentially increasing psychosomatic symptoms. ${ }^{22}$ Daily variations in sleep, or within-person level sleep irregularity, ${ }^{23}$ may reflect daily exposures to work-family conflict or other demands that 
impinge upon the physiologic drive for a regular sleep pattern. Therefore, the third aim of this study was to examine the effects of the intervention on day-to-day variability in employee sleep. We predicted that the intervention would decrease day-to-day variability in employees' nighttime sleep duration, WASO, and daytime nap duration. We also explored age-related differences in these effects.

\section{Methods}

\section{Participants and procedure}

Data came from the Work, Family, and Health Study. ${ }^{9,24}$ The current study used data from a sample of employees working in teams within the IT division of a large Fortune 500 firm. Information technology employees working in the metropolitan areas with the 2 largest worksites were invited to participate in the study. The worksites were selected with the corporate leadership's endorsement to facilitate the study. ${ }^{9}$ Trained field interviewers administered face-to-face computer-assisted personal interviews (CAPIs) to employees at the workplace, beginning in September 2009 and ending in September 2010, to get information on their demographic and work characteristics. Employees who completed a 60minute worksite interview received a $\$ 20$ incentive.

Immediately after the CAPI, interviewers introduced the actigraphy data collection process. If the participant agreed, the interviewer instructed them to wear a sleep monitor (Spectrum; Philips-Respironics, Murrysville, PA) on their nondominant wrist for a week except in situations in which the watch could be damaged (eg, excessive impact, extreme temperatures). Of the total 1171 employees who were invited, 823 employees completed the CAPI interview at baseline (70\% response rate). Of these, 635 employees completed a weeklong actigraphy data collection (baseline). One year later, 569 employees (90\% retention) completed a second week-long actigraphy data collection (12 months). Among 569 employees, 474 provided valid actigraphy for $3+$ days at baseline and 12 months, a commonly used inclusion criterion in prior studies. ${ }^{8}$ In this study, we required a minimum of 5 days for reliable and valid estimation for day-to-day variability of sleep. Thus, the final sample for the current analyses was 396 employees who provided valid actigraphy data for 5 to 10 days at the 2 time points (waves, hereafter). On average, participants had 7 days of valid actigraphy across waves (SD, 0.87). Employees received additional $\$ 20$ for providing their actigraphy sleep data at each wave. Therefore, the total compensation for our study sample was $\$ 60$.

Participants had a mean (SD) age of 46.97 ( \pm 8.45 years). Slightly more than half $(59 \%)$ of the employees were men. Most (80\%) were married or living with permanent romantic partner; $74 \%$ were white and $16 \%$ were Asian or Pacific Islander; $23 \%$ were providing care to their adult parents or relatives; $75 \%$ were college graduates and $22 \%$ had some college (1-3 years). The mean annual household income was in the range of 110,000-119,999. About half (48\%) had children younger than 18 years living in the home. Mean (SD) work hours per week was 46.52 (7.33) hours. Most (79\%) worked with standard dayshift (ie, Monday-Friday, 9-5) and the rest had variable shifts (that changes from day to day, but did not consist of regular evening/night shift workers, per se). 


\section{Workplace intervention}

The Work, Family, and Health Study designed a workplace intervention to increase employee control over their work schedule and increase supervisor and coworker support. ${ }^{9}$ After the baseline CAPI interviews, a total of 56 study groups (aggregation of work teams that operated in the organization) were randomized to either the STAR intervention condition ( $n=195)$ or the control condition $(n=201)$. The STAR intervention consisted of a 3-month structural and cultural change process, including 2 types of main activities: (1) facilitator-led sessions for both employees and managers to help transition them from rigid work schedule to give employees more control over when and where they work and (2) manager-specific training, goal setting, and behavioral self-monitoring focused on increasing family supportive supervisor behavior (for details on STAR procedures, see Kelly et $\mathrm{al}^{10}$ and Kossek et $\mathrm{al}^{25}$ ). Employees who were assigned to the control condition continued business as usual (usual practice) without any entailed activities.

\section{Actigraphic sleep measures}

Actigraphy data came from each participant's actiwatch using the Actiware Sleep Scoring Program (v5.71; Philips-Respironics). We used a standard algorithm recently validated ${ }^{26}$ and at least 2 members of the scoring team determined the validity of each recording based on study-specific standard sleep criteria applied similarly to all recordings. ${ }^{8}$ Each sleep episode was identified if it began at the last epoch of high activity ( $>10$ activity counts) preceding at least five 30 -second epochs of $<10$ activity counts, indicating little to no movement. Possible sleep episodes were determined for low movement count periods by visual inspection and considering changes in light levels (eg, suddenly decreased light levels for that putative nap interval were confirmatory but not required for a nap designation), as well as the overall profile (eg, was the nap similar in low levels of activity to nighttime sleep?). A determination of the actual presence and amount of sleep during those rest intervals was calculated via algorithm (see below). Timing was also considered for categorization of each visually identified sleep periods as either a nighttime (main) sleep period or a daytime nap.

\section{Nighttime sleep duration}

Among sleep episodes, an episode with the longest sleep period of the day was defined as the main, nighttime sleep episode (all other sleep episodes were defined as nap episodes). Daily nighttime sleep duration was computed for main nighttime sleep episodes as the amount of nighttime sleep attained on a day in minutes.

\section{Wake after sleep onset}

Daily WASO was computed as time spent "waking" after sleep onset and before sleep offset during nighttime sleep on a given day, in minutes, as previously validated vs polysomnography. ${ }^{26}$

\section{Daytime nap duration}

Sleep periods coded as nap episodes were summed at the daily level. We initially set the minimum nap rest interval duration at 30 minutes for discrimination of substantive naps from brief periods of no movement. Considering that naps may be rare among daytime 
employees, we also included naps in the range of 25.5-29.5 minutes in the analysis, which was $0.05 \%$ of total naps in our sample (53 naps from 15 employees). Daily nap duration for those who had any naps on a given day was calculated in minutes.

\section{Age and covariates}

Employee age (in 1-year intervals) was tested as a moderator and also included as a covariate. Given that employees' personal and family characteristics may denote family demands and responsibilities as well as their sleep behavior, ${ }^{27,28}$ we controlled for sex $(0=$ female, $1=$ male $)$, marital/partner status $(0=$ unmarried, $1=$ married or living with a partner $)$, living with children younger than 18 years in household $(0=n o, 1=y e s)$, and providing care to adult relatives $(0=n o, 1=y e s)$ in all models. Sociodemographic factors, including race $(0=$ white, $1=$ Asian/Pacific Islander/Other, 2 dummy variables were created) and range of current annual household income ( $1=$ less than 49,999 to $12=$ more than 150,000), were included in all models. Furthermore, to consider possible differences from work characteristics, total work hours assessed by asking "How many hours do you work at any job?" and work schedule $(0=$ standard dayshift, $1=$ variable shifts $)$ were controlled for. In addition, job titles were coded as core ( $=1$; eg, software developers) vs noncore ( $=0$; eg, technical support), following the practice in prior research. ${ }^{21}$ In the IT firm, there was an organizational level merger and about half of employees had not yet received the intervention when merger was announced, whereas others had already received it. To take into account potential differences from this, we included an indicator for the event sequence between merger and intervention $(0=$ Intervention Premerger, $1=$ Intervention Postmerger $)$ in our analysis. Lastly, we controlled for weekend vs weekday effect $(0=$ weekdays, $1=$ weekends; $29 \%$ of the study days were weekends). All continuous variables were centered at sample means.

\section{Data analysis}

We fit multilevel models with SAS Proc Mixed ${ }^{29}$ to take into account the clustered data structure: 5550 daily observations across the 2 waves clustered within 396 employees. There were relatively small variances at the study group level or wave level compared with variances at daily and individual levels, and hence, we used 2-level models to simplify model structure. Because of missing values in covariates, $10 \%$ of observations were not included in analyses; missing cases were not systematically different from nonmissing cases. The model for nap duration was estimated based only on 1448 nap days for employees ( $\mathrm{n}=$ 163) who napped at both waves and had no missing responses on covariates. For the daytime napping models, we included only participants with naps at both waves to evaluate intervention-related changes on total daytime napping duration (but also conducted supplementary analyses with binary nap variable, see "Age differences in intervention effects on changes in daily amounts of nighttime sleep and daytime napping"). Examining changes in nap duration over time enables us to rule out a possibility that the likelihood of napping at one time point is random and nonsystematic.

To examine main intervention effects on changes in daily nighttime sleep duration, WASO, and daytime nap duration, we included a 2-way interaction between the intervention condition and a wave $(0=$ baseline, $1=12$ months $)$. This interaction parameter represents 
the difference in changes in a sleep outcome from baseline to 12 months between STAR employees relative to control employees.

To test whether intervention effects on changes in sleep differed by employee age, a 3-way interaction-intervention $\times$ wave $\times$ age - was included. The 3-way interaction parameter represents whether differences in changes in a sleep outcome from baseline to 12 months between STAR relative to control employees are further differentiated with increasing age. We conducted follow-up tests using estimate commands in Proc Mixed to assess differences between younger employees (38 years, or $-1 \mathrm{SD}$ ) and older employees (56 years, or $+1 \mathrm{SD}$ ) for each intervention condition.

Lastly, to examine intervention effects on changes in day-to-day variability of sleep, we used multilevel models with heterogeneous within-person variance. Daily variance in a sleep outcome was modeled in random effects and estimated by the exponential function (Exp) to use a linear prediction while eliminating the dependence of the variance on the mean level. ${ }^{30}$ Positive coefficients indicate greater day-to-day variability in a sleep outcome. Of note, this approach can estimate more robust variability than the individual SD (iSD) or variance approach because it is model-based and takes into account temporal dependence of data (day effect) as well as the effects of covariates on the mean level. ${ }^{21,31}$ In this approach, however, we cannot test the statistical significance of slopes for an interaction in random effects. Thus, in the case of a significant interaction, we conducted a follow-up test taking quantified individual-level variances across days (iSD ${ }^{2}$ ) as an outcome. By doing so, we may underestimate changes in employees' day-to-day variability by the intervention and/or by age, because there is less power to detect effects when using quantified variance estimates compared with when modeling variability. ${ }^{31}$

\section{Results}

\section{Descriptive results}

Demographic and work characteristics of our sample of IT employees, with actigraphic sleep measures at baseline, are presented in Table 1. We also present results from difference tests (analysis of variance, $\chi^{2}$ tests) by employee age, divided into 3 age groups based on mean age \pm 1 SD at baseline: younger, 24 to 38 years $(n=72)$; middle-aged, 39 to 55 years $(n=257)$; or older employees, 56 to 70 years $(n=67)$. On average, employees had 424 minutes (7 hours) of nighttime sleep duration (SD, 54.8) and 41 minutes of WASO (SD, 16.6), per day, with no statistical difference by age groups.

In terms of daytime napping, 253 employees (64\%) had any naps at baseline. Among those with any naps at baseline (Table 1), 22\% of their sleep episodes were naps, and they had 2.2 naps, on average, across the study days (SD, 1.6) and 1.2 naps on days when they napped (median, 1 nap; about 19\% of nap days included 2 naps on average for that day). For those who had any naps, the mean (SD) daytime napping across total study days was 23 (21.7) minutes and the mean (SD) daytime napping across only days when they nap was 67 (38.2) minutes (median, 57.5 minutes) at baseline. Thus, two-thirds of our sample napped for less than 30 minutes across all days in a week of collection, and for about an hour on a nap day (that happened on average twice a week). The model for nap duration included 1448 nap 
days in participants who napped at both waves and had no missing responses on covariates ( $n=163$ employees; $41 \%$ of the sample). These nap days occupied about $30 \%$ of total study days used in the analyses (Table 2).

Although age was not related to having naps, younger employees had longer naps than did middle-aged and older employees. To provide proxy estimates for day-to-day variability in sleep measures, we included individual SDs (iSDs) of each sleep variable. The degrees of daily variation were 64,14 , and 32 minutes around person means for nighttime sleep, WASO, and nap duration, respectively. There were no age differences in these iSDs of sleep measures. Greater iSD of nighttime sleep duration was associated with longer nap duration $(r=0.18, P<.01)$ and greater iSD of WASO $(r=0.27, P<.001)$.

\section{Main effects of the intervention on changes in daily amounts of nighttime sleep and daytime napping}

Table 2 shows results from multilevel models separately predicting daily nighttime sleep duration, WASO, and nap duration. In Model 1, there was a significant interaction between wave and intervention predicting nighttime sleep duration: intervention employees increased nighttime sleep duration ( 3 minutes more per day) but control employees decreased (6 minutes less per day), resulting in a significant 9-minute difference in daily nighttime sleep duration between the 2 groups (Fig. 1, panel 1). This result is consistent with previous intervention effects for 24-hour total sleep duration. ${ }^{8}$ There were no significant interactions between wave and intervention predicting changes in the amount of WASO or nap duration.

\section{Age differences in intervention effects on changes in daily amounts of nighttime sleep and daytime napping}

In Model 2 of Table 2, we tested whether intervention effects differed by employee age. There was a significant 3-way interaction between age, wave, and intervention predicting nap duration (but no effects on nighttime sleep duration or WASO). Panel 2 in Fig. 1 shows that the difference in nap duration between older employees (+1 SD corresponds to 56 years) in the intervention condition and those in the control was significant with a difference of 17 minutes less per day. Specifically, control older employees increased nap duration, but intervention older employees did not. Younger employees ( -1 SD corresponds to 38 years) in the intervention condition did not significantly change nap duration compared with control younger employees. We also conducted supplementary analyses with binary nap variable (napping vs no napping) that includes those who napped at only one wave. Neither the main effect of the intervention nor age differences in the intervention effect on changes in the likelihood of napping were significant.

\section{Age differences in intervention effects on changes in daily variability of nighttime sleep and daytime napping}

Table 3 shows results from heterogeneous within-person variance models predicting changes in day-to-day variability in nighttime sleep duration, WASO, and nap duration. There was no significant interaction between age, wave, and intervention predicting daily variance of nighttime sleep duration. However, significant 3-way interaction effects emerged for daily variance of WASO and nap duration. To interpret the interaction effect on daily variability in 
WASO, we conducted a follow-up analysis predicting individual variance of WASO with the same set of predictors (individual mean levels of WASO were controlled). The 3-way interaction also predicted individual variance of WASO at a trend level $(B=-8.56, \mathrm{SE}=$ $4.56, P=.062)$. The post-hoc test indicated that older employees in the intervention condition significantly decreased day-to-day variability in WASO at 12 months compared with their baseline- 90 minutes $^{2}$ less variance or 9.5 minutes less SD around person means (Fig. 1, panel 3). Older employees in the control condition did not significantly change daily variability of WASO and the difference in variability of WASO between the 2 groups did not reach statistical significance. Lastly, with regard to daily variability in nap duration, younger employees in the intervention condition exhibited an increase in daily variability of nap duration at 12 months relative to their baseline.

\section{Discussion}

This study examined the effects of a workplace intervention on changes in several dimensions of employee sleep assessed by actigraphy and whether the effects differed by employee age. Our findings demonstrate that the workplace intervention, previously established as effective for decreasing work-to-family conflict ${ }^{10}$ and increasing overall (24hour) total sleep time, ${ }^{8}$ was specifically efficacious for increasing nighttime sleep duration but not daytime nap duration. Compared with the control group, intervention employees had greater nighttime sleep duration by 63 minutes per week ( 9 minutes per day $\times 7$ days). The magnitude of this effect was almost identical with the effect on total sleep duration, ${ }^{8}$ but suggests a stronger effect of the intervention during nighttime sleep episodes. Moreover, the effects of the intervention on daytime nap duration and daily variability of WASO differed by employee age. The intervention decreased older employees' daytime nap duration and day-today variability in WASO. This study contributes to understanding on how a workplace flexibility/support intervention can change employees' nighttime sleep and daytime napping differently by age. This study also advances the methodology examining sleep by assessing both the amount and day-to-day variability of sleep.

This study provides evidence that the workplace intervention was effective for specifically improving employees' nighttime sleep quantity. Employees randomly assigned to the intervention condition significantly had greater nighttime sleep duration at 12 months, relative to employees in the control condition (who decreased nighttime sleep duration). This result extends the previous finding ${ }^{8}$ that STAR increased total sleep duration-across nighttime and daytime - and perceived sleep sufficiency. Previous studies showed that the STAR intervention significantly decreased work-to-family conflict, burnout, perceived stress, and psychological distress among IT employees. ${ }^{10,32}$ Together, a possible mechanism of how the intervention was effective in improving employee sleep may be that those who received the intervention were able to reduce the extent that work stressors interfere with their recovery in the personal domain, and thus increased their nighttime sleep duration. The intervention effect on changes in nighttime sleep duration was found regardless of employee age, sex, family background, and even work hours, all important predictors of sleep. ${ }^{27,28}$ Our rigorous study design—randomization to treatment and objective markers of sleepalso enhances our confidence in interpreting the positive intervention effect on increasing employees' nighttime sleep duration. Considering that the average sleep duration at baseline 
was already generally sufficient in our sample (mean, 7.6 hours; Table 2), the effect size of 9 minutes difference per day may be a conservative one-there may be a larger effect of the intervention on increasing nighttime sleep duration for a different sample with a greater proportion obtaining insufficient sleep. Note that there was no intervention effect on the amount of nighttime WASO (previous research ${ }^{8}$ also reported no intervention effect on total WASO). A potential explanation for this null effect is that, unlike nighttime sleep duration, the amount of WASO is less likely to be changed by external factors. It may be more likely to be affected by employees' physiological factors the present study did not address.

This study found older employees receiving the workplace intervention decreased day-today variability in WASO at 12 months compared with their baseline. Greater variability in WASO refers to greater irregularity in nighttime sleep quality, ${ }^{21}$ which can be interpreted as a greater degree of daily fluctuation from his/her average sleep quality. Although longer WASO reflects poorer sleep quality, the person-level mean of WASO may indicate the individualized level of sleep quality (whether greater or lower). ${ }^{33,34}$ Hence, that older employees in the intervention exhibited a decrease in daily fluctuation of WASO suggests that the intervention allowed older employees to regularly maintain nighttime sleep quality. There was no significant change for younger employees in the intervention condition. Given prior research that documents greater frequency and duration of WASO among older individuals, ${ }^{16-18}$ intervention effects might have been more pronounced for older employees who had more WASO compared with the other age groups (although the baseline difference was not statistically significant; Table 1). The intervention effect on older employees' decreased variability of WASO demonstrates within-person decreases in variability of sleep quality at postintervention compared with baseline (whether greater or smaller). Note, however, that younger and middle-aged employees in the control condition also exhibited significant decreases in daily variability of WASO at 12 months compared with their baseline. Given that stress is tightly linked to variability of sleep fragmentation, ${ }^{35}$ some unknown stressors confounded with younger and middle-aged control employees' variability in WASO might have dissipated over the 12 months and thus decreased their variability of WASO. Further analyses are needed to understand additional factors contributing to their decreases in day-to-day variability in WASO even without receiving the intervention.

The effects of the STAR intervention on daytime nap duration also differed by age. Older employees in the intervention condition significantly decreased nap duration at 12 months relative to older employees in the control condition, whereas younger employees in the intervention condition did not. Prior studies showed older adults nap more frequently (and perhaps longer) than do their younger counterparts. ${ }^{19,20}$ Decreases in sleep efficiency across time may contribute to such age-related differences in napping. However, in our study, the intervention protected older employees from increasing nap duration. Even brief naps can have salutary benefits, but longer naps (eg, longer than about a half hour) can produce sleep inertia, a persistence of post-sleep grogginess upon awakening. ${ }^{36}$ Our sample of employees who had any naps took naps about 67 minutes per day at baseline (the intercept of daily nap duration in Table 2). This means that for those who needed/took naps, they all took a sufficient amount of naps. However, the nap duration of older employees who were not in the intervention condition increased even more at 12 months than their baseline (panel 2, Fig. 1). Given that nighttime sleep duration decreased for older employees who did not get 
the treatment, they might have experienced an increased need for napping to counteract their nighttime sleep loss. In contrast, employees randomized to treatment tended to increase nighttime sleep duration and, further, those who were older also decreased day-to-day variability in WASO, and thus might not have such increased need for napping. In addition, in our supplementary analyses, we did not find either the main effect of the intervention or the interaction between the intervention and age predicting changes in the likelihood of napping (vs no napping). By examining daily nap duration that varies within an employee, this study found that the workplace flexibility/support intervention protected older employees from increasing nap duration. Taken together, our findings suggest that older employees randomized to the intervention improved nighttime sleep quantity and regularity in sleep quality and, by doing so, mitigated their need for daytime napping to recover from inadequate nightly sleep.

The sample was purposively selected from an IT firm and, thus, a limitation is that findings may not generalize to employees in other contexts. Our sample of white-collar and professional employees is relatively privileged, which may be related to a lack of a significant main effect of age on sleep, such as shorter nighttime sleep, longer WASO, and more napping for older than younger employees seen in other studies. ${ }^{16-20,37}$ Also note that those in other work contexts may not be able to take a nap in the middle of their work day, as our sample of employees did. In our sample, $41 \%$ of employees napped for more than an hour on days when they napped, averaged about 2 naps/wk, and these occurred on about $30 \%$ of total valid actigraphy days. Future research should compare these napping patterns with different industry samples. Future research may also need to test whether and how a workplace intervention can improve employee sleep across ages in other industry samples.

In conclusion, we find positive workplace intervention effects on increasing employees' nighttime sleep duration, and for older employees on decreasing daytime nap duration and day-to-day variability in WASO. Future workplace interventions could be tailored based on moderators like age to more powerfully impact sleep outcomes. Sleep is important not only for employee-level well-being and health, but also for organizational-level productivity. ${ }^{38,39}$ Workplaces should continue to make efforts to transform their work practices to reduce the extent that work interferes with employee sleep in nonwork domain, which, in turn, will bring positive returns on their investment.

\section{References}

1. Centers for Disease Control and Prevention. Short sleep duration among workers-United States, 2010. MMWR Morb Mortal Wkly Rep. 2012; 61(16):281-285. [PubMed: 22534760]

2. Watson NF, Badr MS, Belenky G, et al. Joint consensus statement of the American Academy of Sleep Medicine and Sleep Research Society on the recommended amount of sleep for a healthy adult: methodology and discussion. Sleep. 2015; 38(8):1161-1183. http://dx.doi.org/10.5664/jcsm. 4950. [PubMed: 26194576]

3. Mischel, L. Vast majority of wage earners are working harder, and for not much more: trends in U.S work hours and wages over 1979-2007. In: Mishel, L.; Bivens, J.; Gould, E.; Shierholz, H., editors. The state of working America. 12. Ithaca, N.Y: Cornell University Press; 2013. [Supplement]

4. Barnes CM, Wagner DT, Ghumman S. Borrowing from sleep to pay work and family: expanding time-based conflict to the broader nonwork domain. Pers Psychol. 2012; 65(4):789-819. http:// dx.doi.org/10.1111/peps.12002. 
5. Rosekind MR, Gregory KB, Mallis MM, Brandt SL, Seal B, Lerner D. The cost of poor sleep: workplace productivity loss and associated costs. J Occup Environ Med. 2010; 52(1):91-98. http:// dx.doi.org/10.1097/JOM.0b013e3181c78c30. [PubMed: 20042880]

6. Moen P, Kelly EL, Tranby E, Huang Q. Changing work, changing health: can real work-time flexibility promote health behaviors and well-being? J Health Soc Behav. 2011; 52(4):404-429. [PubMed: 22144731]

7. Moen P, Fan W, Kelly EL. Team-level flexibility, work-home spillover, and health behavior. Soc Sci Med. 2013; 84:69-79. http://dx.doi.org/10.1016/j.socscimed.2013.02.011. [PubMed: 23517706]

8. Olson R, Crain TL, Bodner TE, et al. A workplace intervention improves sleep: results from the randomized controlled work, family, and health study. Sleep Heal. 2015; 1(1):55-65. http:// dx.doi.org/10.1016/j.sleh.2014.11.003.

9. Bray, J.; Kelly, E.; Hammer, L., et al. An integrative, multilevel, and transdisciplinary research approach to challenges of work, family, and health; Methods Res RTI Press. 2013. p. 1-38.http:// dx.doi.org/10.3768/rtipress.2013.mr.0024.1303

10. Kelly EL, Moen P, Oakes JM, et al. Changing work and work-family conflict: evidence from the work, family, and health network. Am Sociol Rev. 2014; 79(3):485-516. http://dx.doi.org/ 10.1177/0003122414531435. [PubMed: 25349460]

11. Lovato, N.; Lack, L. The effects of napping on cognitive functioning. In: Kerkhof, GA.; Van Dongen, HPA., editors. Progress in Brain Research. Vol. 185. Elsevier B.V; 2010. p. 155-166.http://dx.doi.org/10.1016/B978-0-444-53702-7.00009-9

12. Bliwise, DL.; Scullin, MK. Normal Aging. In: Kryger, MH.; Roth, T.; Dement, WC., editors. Principles and Practice of Sleep Medicine. Philadelphia, PA: Elsevier; 2017. p. 25-38.

13. Reilly T, Waterhouse J, Atkinson G. Aging, rhythms of physical performance, and adjustment to changes in the sleep-activity cycle. Occup Environ Med. 1997; 54:812-816. http://dx.doi.org/ 10.1136/oem.54.11.812. [PubMed: 9538354]

14. Ilmarinen J. Ageing workers in Finland and in the European Union: their situation and the promotion of their working ability, employability and employment. Geneva Pap Risk Insur. 2001; 26(4):623-641. http://dx.doi.org/10.1111/1468-0440.00144.

15. Kiss P, De Meester M, Braeckman L. Differences between younger and older workers in the need for recovery after work. Int Arch Occup Environ Health. 2008; 81(3):311-320. http://dx.doi.org/ 10.1007/s00420-007-0215-y. [PubMed: 17576592]

16. Ohayon MM, Carskadon MA, Guilleminault C, Vitiello MV. Meta-analysis of quantitative sleep parameters from childhood to old age in healthy individuals: developing normative sleep values across the human lifespan. Sleep. 2004; 27(7):1255-1273. [PubMed: 15586779]

17. Floyd JA, Medler SM, Ager JW, Janisse JJ. Age-related changes in initiation and maintenance of sleep: a meta-analysis. Res Nurs Health. 2000; 23(2):106-117. http://dx.doi.org/10.1002/ (SICI) 1098-240X(200004)23:2<106::AID-NUR3>3.0.CO;2-A. [PubMed: 10782869]

18. Hoch CC, Dew MA, Reynolds III, Monk TH. A longitudinal study of laboratory- and diary-based sleep measures in healthy "old old" and "young old" volunteers. Sleep. 1994; 17(6):489-496. [PubMed: 7809561]

19. National Sleep Foundation. Sleep in America Poll. 2003; 2003 [http://www.sleepfoundation.org].

20. Ohayon MM, Zulley J. Prevalence of naps in the general population. Sleep Hypn. 1999; 1(2):8897.

21. Buxton OM, Lee S, Beverly C, et al. Work-family conflict and employee sleep: evidence from IT workers in the Work, Family and Health Study. Sleep. 2016; 39(10):1871-1882. [PubMed: 27568810]

22. Nagane M, Suge R, Watanabe S-I. Relationship between psychosomatic complaints and circadian rhythm irregularity assessed by salivary levels of melatonin and growth hormone. J Circadian Rhythms. 2011; 9(1):9. http://dx.doi.org/10.1186/1740-3391-9-9. [PubMed: 21914213]

23. Zee PC, Badr MS, Kushida C, et al. Strategic opportunities in sleep and circadian research: report of the joint task force of the Sleep Research Society and American Academy of Sleep Medicine. Sleep. 2014; 37:219-227. http://dx.doi.org/10.5665/sleep.3384. [PubMed: 24501434] 
24. King, RB.; Karuntzos, G.; Casper, LM., et al. Work-family balance issues and work-leave policies. In: Gatchel, RJ.; Schultz, IZ., editors. Handbook of Occupational Health and Wellness. New York, NY: Springer; 2013. p. 323-340.

25. Kossek EE, Hammer LB, Kelly EL, Moen P. Designing work, family \& health organizational change initiatives. Organ Dyn. 2014; 43:53-63. http://dx.doi.org/10.1016/j.orgdyn.2013.10.007. [PubMed: 24683279]

26. Marino M, Li Y, Rueschman MN, et al. Measuring sleep: accuracy, sensitivity, and specificity of wrist actigraphy compared to polysomnography. Sleep. 2013; 36(11):1747-1755. http://dx.doi.org/ 10.5665/sleep.3142. [PubMed: 24179309]

27. Burgard SA. The needs of others: gender and sleep interruptions for caregivers. Soc Forces. 2011; 89(4):1189-1215. http://dx.doi.org/10.1093/sf/89.4.1189.

28. Maume DJ, Sebastian RA, Bardo AR. Gender, work-family responsibilities, and sleep. Gend Soc. 2010; 24(6):746-768. http://dx.doi.org/10.1177/0891243210386949.

29. Littell, RC.; Stroup, WW.; Milliken, GA.; Wolfinger, RD.; Schabenberger, O. SAS for Mixed Models. SAS institute; 2006.

30. Hoffman L. Multilevel models for examining individual differences in within-person variation and covariation over time. Multivar Behav Res. 2007; 42(4):609-629. http://dx.doi.org/ 10.1080/00273170701710072.

31. Wiley JF, Bei B, Trinder J, Manber R. Daily sleep variability quantified: a reliable and flexible approach. Sleep. 2015; 38(Suppl A136)

32. Moen P, Kelly EL, Fan W, et al. Does a flexibility/support organizational initiative improve hightech employees well-Being? Evidence from the Work, Family, and Health Network. Am Sociol Rev. 2016; 81(1):134-164. http://dx.doi.org/10.1177/0003122415622391.

33. McCurry SM, Gibbons LE, Logsdon RG, Vitiello M, Teri L. Training caregivers to change the sleep hygiene practices of patients with dementia: The NITE-AD project. J Am Geriatr Soc. 2003; 51(10):1455-1460. http://dx.doi.org/10.1046/j.1532-5415.2003.51466.x. [PubMed: 14511168]

34. Berger AM, Kuhn BR, Farr LA, et al. Behavioral therapy intervention trial to improve sleep quality and cancer-related fatigue. Psychooncology. 2009; 6(18):634-646. http://dx.doi.org/10.1002/pon. 1438.

35. Mezick EJ, Matthews KA, Hall M, et al. Intra-individual variability in sleep duration and fragmentation: associations with stress. Psychoneuroendocrinology. 2009; 34(9):1346-1354. http://dx.doi.org/10.1016/j.psyneuen.2009.04.005. [PubMed: 19450933]

36. Milner CE, Cote KA. Benefits of napping in healthy adults: impact of nap length, time of day, age, and experience with napping. J Sleep Res. 2009; 18(2):272-281. http://dx.doi.org/10.1111/j. 1365-2869.2008.00718.x. [PubMed: 19645971]

37. Gangwisch JE, Heymsfield SB, Boden-Albala B, et al. Short sleep duration as a risk factor for hypertension: analyses of the first National Health and Nutrition Examination Survey. Hypertension. 2006; 47(5):833-839. http://dx.doi.org/10.1161/01.HYP.0000217362.34748.e0. [PubMed: 16585410]

38. Barnes CM, Ghumman S, Scott BA. Sleep and organizational citizenship behavior: the mediating role of job satisfaction. J Occup Health Psychol. 2012; 18(1):16-26. http://dx.doi.org/10.1037/ a0030349. [PubMed: 23148600]

39. Barnes CM. Working in our sleep: sleep and self-regulation in organizations. Organ Psychol Rev. 2012; 2(3):234-257. http://dx.doi.org/10.1177/2041386612450181. 


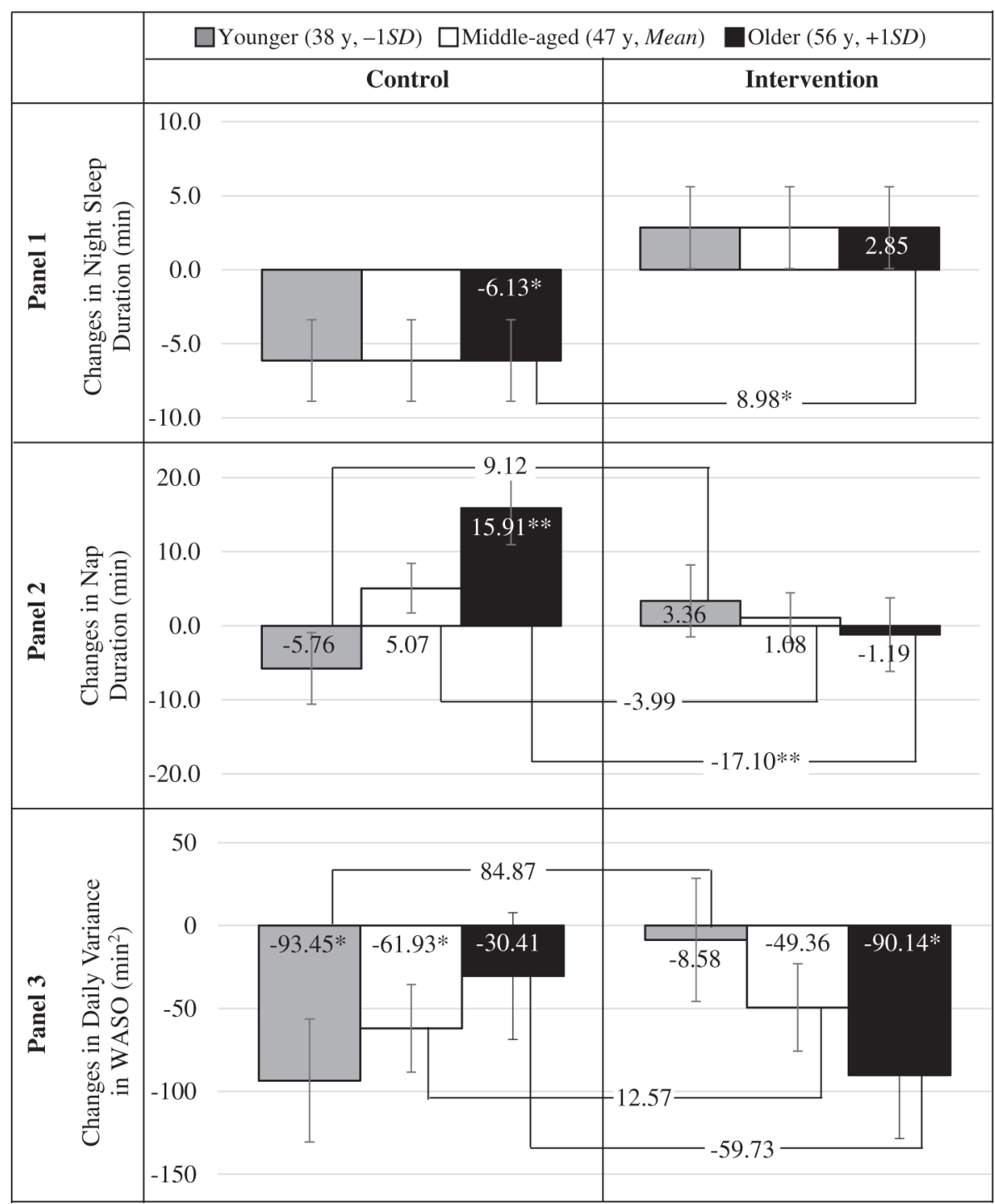

Fig. 1.

The effects of the STAR workplace intervention on nighttime sleep duration, nap duration, and day-to-day variability in WASO. STAR, Support-Transform-Achieve-Results; WASO, wake after sleep onset. $* P<.05, * * P<.01$. 


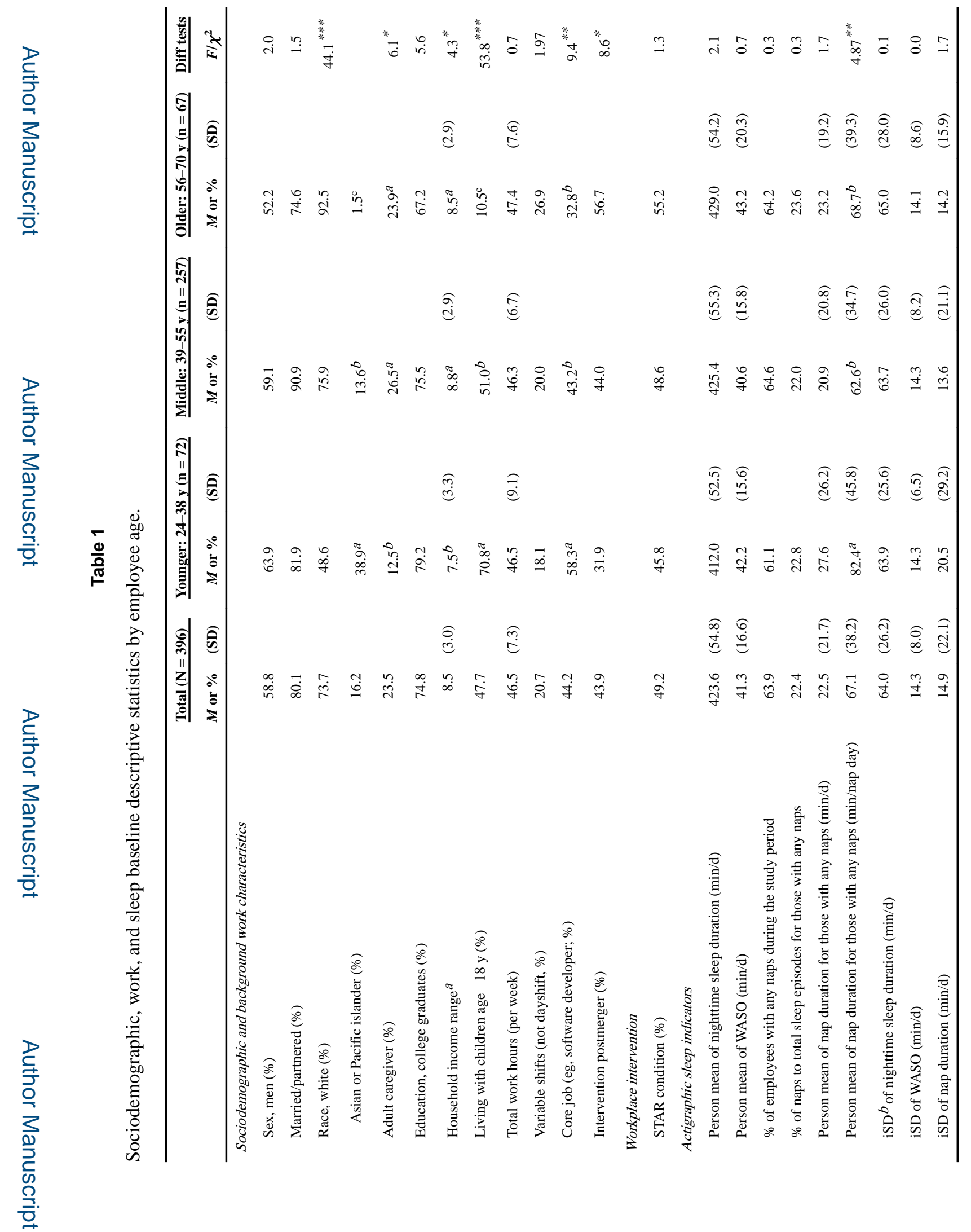

Sleep Health. Author manuscript; available in PMC 2017 January 17. 


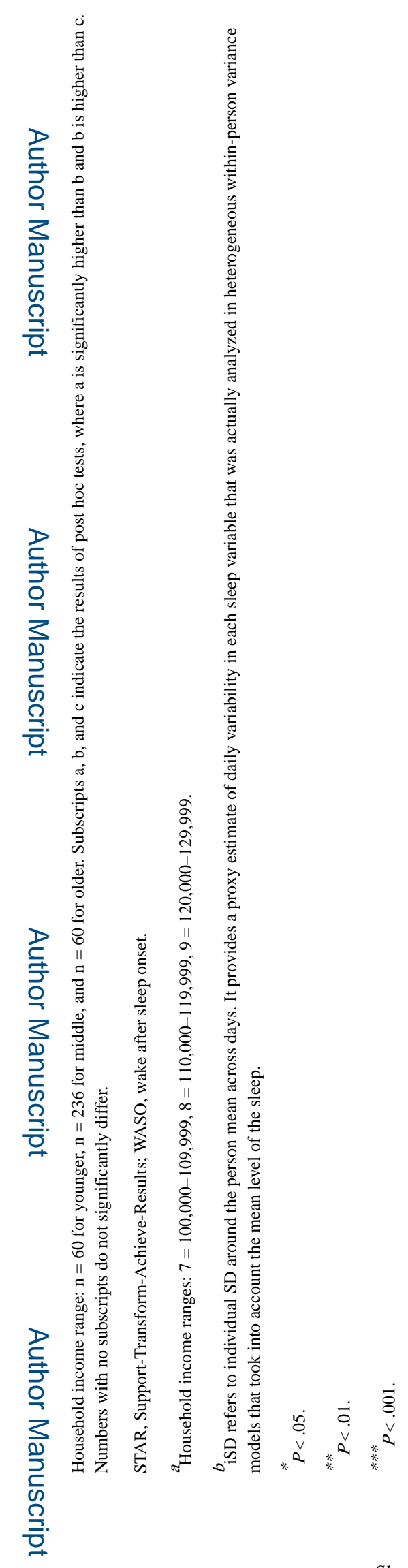

Sleep Health. Author manuscript; available in PMC 2017 January 17. 


\section{Table 2}

Results from multilevel models predicting the levels of daily nighttime sleep duration, wake after sleep onset, and daytime nap duration.

\begin{tabular}{|c|c|c|c|}
\hline & $\begin{array}{l}\text { Daily nighttime sleep } \\
\text { duration (min), } B \text { (SE) }\end{array}$ & $\begin{array}{c}\text { Daily wake after night sleep } \\
\text { onset (min), } B \text { (SE) }\end{array}$ & $\underset{(\mathrm{SE})}{\text { Daily nap duration (min), } B}$ \\
\hline \multicolumn{4}{|l|}{ Fixed effects } \\
\hline \multicolumn{4}{|l|}{ Model 1} \\
\hline Intercept, adjusted mean & $457.52(8.42)^{* * *}$ & $43.69(2.48)^{* * * *}$ & $66.66(5.92)^{* * *}$ \\
\hline Age & $-0.47(0.38)$ & $-0.01(0.11)$ & $-0.57(0.27)^{*}$ \\
\hline Sex, men (vs women) & $-26.20(5.70)^{* * *}$ & $-1.24(1.70)$ & $-2.73(4.02)$ \\
\hline Marital status, married (vs unmarried) & $-11.93(8.05)$ & $-2.80(2.40)$ & $-5.46(5.39)$ \\
\hline Race, Asian/Pacific Islander (vs white) & $-26.89(7.94)^{* * *}$ & $10.78(2.37)^{* * *}$ & $2.80(5.62)$ \\
\hline Race, other (vs white) & $-28.17(9.03)^{* *}$ & $3.04(2.69)$ & $4.27(5.94)$ \\
\hline Adult caregiving, caregiver (vs not) & $3.06(4.03)$ & $-1.66(1.00)^{\dagger}$ & $-3.90(3.81)$ \\
\hline Household income range & $1.04(1.02)$ & $-0.10(0.30)$ & $-0.62(0.68)$ \\
\hline Living with children age $\leq 18$ y (vs not) & $0.77(6.11)$ & $-2.57(1.82)$ & $-6.10(4.20)$ \\
\hline Total work hours & $-0.57(0.25)^{*}$ & $-0.04(0.06)$ & $-0.33(0.26)$ \\
\hline Variable shifts (vs dayshift) & $-1.93(3.64)$ & $1.68(0.89)^{\dagger}$ & $-2.34(3.96)$ \\
\hline Job, core (vs noncore) & $3.01(5.67)$ & $-0.88(1.69)$ & $1.14(3.96)$ \\
\hline Intervention postmerger (vs premerger) & $-5.22(5.43)$ & $-0.77(1.62)$ & $-1.35(3.77)$ \\
\hline Weekends (vs weekdays) & $18.86(2.18)^{* * *}$ & $2.27(0.51)^{* * *}$ & $12.45(2.37)^{* * *}$ \\
\hline Wave, 12 mo (vs baseline) & $-6.13(2.76)^{*}$ & $-2.04(0.64)^{* * *}$ & $4.84(3.56)$ \\
\hline Intervention (vs control) & $-12.72(5.60)^{*}$ & $0.84(1.63)$ & $10.01(4.29)^{*}$ \\
\hline Wave $\times$ intervention & $8.98(3.98)^{*}$ & $1.03(0.93)$ & $-4.08(4.57)$ \\
\hline \multicolumn{4}{|l|}{ Model 2} \\
\hline Age $\times$ wave & $-0.59(0.33)^{\dagger}$ & $-0.10(0.08)$ & $1.28(0.42)^{* *}$ \\
\hline Age $\times$ intervention & $-0.61(0.68)$ & $-0.11(0.20)$ & $0.74(0.53)$ \\
\hline Age $\times$ wave $\times$ intervention & $0.76(0.48)$ & $0.06(0.11)$ & $-1.55(0.57)^{* *}$ \\
\hline \multicolumn{4}{|l|}{ Random effects (for model 2) } \\
\hline Between-person level variance & $2036.47(183.06)^{* * *}$ & $193.81(16.22)^{* * *}$ & $548.62(79.65)^{* * * *}$ \\
\hline Within-person level variance & $4816.20(100.27)^{* * *}$ & $260.91(5.43)^{* * *}$ & $1502.24(62.35)^{* * *}$ \\
\hline
\end{tabular}

A total of 5550 observations were nested within 396 employees; 4981 observations were used in the models for nighttime sleep duration and wake after night sleep onset because of missing values in covariates. The model for nap duration was estimated based only on 1448 nap days for employees who napped at both waves and with no missing responses on covariates $(n=163)$. Primary variables of interest are bolded.

$$
\begin{gathered}
{ }^{\dagger} P<.10 . \\
{ }^{*} P<.05 . \\
{ }^{* *} P<.01 . \\
{ }^{* * *} P<.001 .
\end{gathered}
$$




\section{Table 3}

Results from heterogeneous within-person variance models predicting day-to-day variability in nighttime sleep duration, wake after sleep onset (WASO), and daytime nap duration.

\begin{tabular}{lccc}
\hline & $\begin{array}{c}\text { Day-to-day variability in } \\
\text { nighttime sleep duration } \\
\text { (variance in minutes), } \boldsymbol{a} \text { (SE) }\end{array}$ & $\begin{array}{c}\text { Day-to-day variability in } \\
\text { WASO (variance in minutes), } \\
\boldsymbol{a}(\mathbf{S E})\end{array}$ & $\begin{array}{c}\text { Day-to-day variability in nap } \\
\text { duration (variance in } \\
\text { minutes), } \boldsymbol{a} \text { (SE) }\end{array}$ \\
\hline Random effects & $2030.85(182.84)^{* * *}$ & $196.82(16.39)^{* * *}$ & $582.08(80.26)^{* * *}$ \\
Between-person level variance & $4310.28(180.42)^{* * *}$ & $321.06(13.76)^{* * *}$ & $877.55(83.32)^{* * *}$ \\
Residual variance & $56,964.6$ & $42,625.4$ & $14,783.0$ \\
-2 Res log likelihood & $-0.01(0.00)$ & $-0.01(0.01)^{*}$ & $-0.05(0.01)^{* * *}$ \\
Exp (age) & $0.12(0.06)^{*}$ & $-0.40(0.06)^{* * *}$ & $0.08(0.14)$ \\
Exp (wave) & $0.15(0.06)^{*}$ & $-0.13(0.06)^{*}$ & $0.50(0.14)^{* * *}$ \\
Exp (Intervention) & $-0.12(0.09)$ & $0.16(0.09)$ & $0.41(0.20)$ \\
Exp (wave $\times$ intervention) & $-0.00(0.01)$ & $0.01(0.01)$ & $0.12(0.02)^{* * * *}$ \\
Exp (age $\times$ wave) & $0.01(0.01)^{*}$ & $0.03(0.01)^{* * *}$ & $0.06(0.02)^{* * *}$ \\
Exp (age $\times$ intervention) & $-0.00(0.01)$ & $-0.04(0.01)^{* * *}$ & $-0.16(0.03)^{* * *}$ \\
Exp (age $\times$ wave $\times$ intervention) & & &
\end{tabular}

A total of 5550 observations were nested within 396 employees; 4981 observations were used in the models for nighttime sleep duration and wake after night sleep onset because of missing values in covariates. All covariates from the previous models, such as employee age, sex, marital/partner status, race/ethnicity, adult caregiver status, range of current annual household income, presence of children younger than 18 years in household, total work hours, occupation, merger indicator, and weekend, were included in the fixed effects to control for the effects on the mean levels of nighttime sleep duration, WASO, and nap duration. The model for nap duration was estimated based only on 1488 nap days for employees who napped at both waves and with no missing responses on covariates $(n=163)$. Primary variables of interest are bolded.

*

${ }^{*} P<.05$.

**** $P<.001$. 